\title{
Resolving Ambiguities in Confused Online Tamil Characters with Post Processing Algorithms
}

\author{
A.G. Ramakrishnan and Suresh Sundaram \\ Medical Intelligence and Language Engineering Laboratory \\ Indian Institute of Science, Bangalore, India \\ \{ramkiag, suresh\}@ee.iisc.ernet.in
}

\begin{abstract}
This paper addresses the problem of resolving ambiguities in frequently confused online Tamil character pairs by employing script specific algorithms as a post classification step. Robust structural cues and temporal information of the preprocessed character are extensively utilized in the design of these algorithms. The methods are quite robust in automatically extracting the discriminative sub-strokes of confused characters for further analysis. Experimental validation on the IWFHR Database indicates error rates of less than $3 \%$ for the confused characters. Thus, these post processing steps have a good potential to improve the performance of online Tamil handwritten character recognition.
\end{abstract}

Keywords: Confusion Pairs, Sub stroke Extraction and analysis, Fourier Descriptors, Online handwritten character recognition.

\section{Introduction}

Tamil is a popular classical language spoken by a significant population in South East Asian countries. There are 156 distinct symbols in Tamil [1]. As far as earlier work on recognition of online Tamil characters is concerned, Deepu et al. [2] generate class specific subspaces using principal component analysis, while Niranjan et al. [1] have employed dynamic time warping for matching unequal length feature sequences. Hidden Markov models for recognition have also been reported in [3] [4]. In a recent work, we have studied the performance of the 2DPCA Algorithm [5], which was originally proposed for face recognition.

Each of the above schemes is found to give nearly similar generalization performances on a given test data. Most of the misclassifications of the given data, in general, are attributed to the fact that Tamil has many symbols that look visually similar. Any classifier that works on features at a global level fails to capture finer nuances that make these symbols distinct. One way to circumvent this drawback would be to incorporate a post processing step that employs local features to reduce the degree of confusion between frequently confused characters, and thereby improves the overall performance of the recognition. Specifically, this paper proposes algorithms for disambiguating frequently confused symbols. The approaches are developed, taking into account, the popular writing / lexemic styles of modern Tamil script. They can be applied irrespective of the nature of the classifier used for the recognition. 
In a system that deals with recognition at the word level one could use language models. However, when recognizing isolated characters, one is devoid of any such additional information that can be used to correct errors. Thus, we resort to the use of robust structural features for post recognition disambiguation.

\section{Confusion Pair Analysis}

A careful analysis of the confusion matrices, obtained with different classifier frameworks, suggests that the frequently confused Tamil characters can be manually grouped into two categories A and B [5]. Accordingly, we propose appropriate postprocessing techniques to each group of the confusion pairs. The confusions in Group A appear between pairs of Tamil consonant-vowel combinations sharing the same base consonant but different vowel modifiers. The confused strokes contributing to errors are $\Omega$ and $\odot$. These correspond to the modifiers of the vowels $\&$ and $\%$ respectively.

Apart from Group A pairs, there exist other pairs that differ predominantly in the

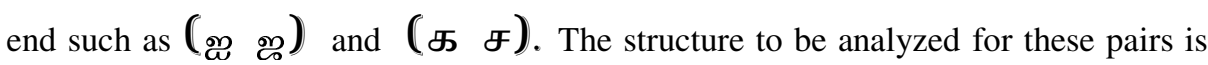
strikingly different from those belonging to Group A. Consequently, these pairs are placed in Group B. Also, there exist certain character pairs that differ only at the start and middle of the trace such as (ஏ rated in Group B. As stated before, the spatial and temporal information provided by the online data is extensively utilized for the design of the algorithms. Prior to designing the appropriate post processing technique, the raw Tamil character is subject to pre processing modules [2] such as smoothing, size normalization and resampling.

\section{Disambiguation of Group A Confusion Pairs}

In this section, we outline the post processing algorithm for disambiguating the vowel modifiers $q$ and $\odot$ for a given base consonant. Popular writing styles of modern Tamil script suggest that the vowel modifiers 0 and $\odot$ always form the last stroke in any multistroke consonant-vowel combination character. However, for CV combinations written as a single stroke (where the vowel modifiers are written as a continuation of the base consonant), a subset of sample points, carefully chosen before the final PEN UP signal, is taken to be the vowel modifier. It is worth reemphasizing that the confused pairs in Group A correspond to CV combinations sharing the same base consonant $(\mathrm{BC})$. Let $\omega_{1}$ and $\omega_{2}$ denote the class labels of $\mathrm{BC}+9$ and $\mathrm{BC}+\odot$ combinations, respectively. We outline below the algorithm proposed for distinguishing $\omega_{1}$ and $\omega_{2}$. Note that as soon as any 'If' condition in the algorithm is satisfied, the corresponding class label is assigned to the $\mathrm{CV}$ combination and we terminate. 
For the preprocessed $\mathrm{CV}$ combination resampled to $N$ points, let $S=\left\{\left(x_{i}, y_{i}\right)\right\}_{i=b}^{N}$ denote the pen coordinates of the extracted vowel modifier. Here $\left(x_{b}, y_{b}\right)$ denotes the starting sample point of the vowel modifier. A point $\left(x_{i}, y_{i}\right)$ in $S$ is said to be an 'interest point' if the following two conditions are satisfied.

(i) $y_{i}<y_{i-1}$ and $y_{i}<y_{i+1}$.

(ii) $x_{i+1}<x_{i}$.

Using the aforementioned condition, compute the number of interest points $I$.

Find the sample point $\left(x_{s}, y_{s}\right)$ satisfying the relation $y_{s}=\max _{i>b} y_{i}$.

If $\left(\begin{array}{ll}x_{s} & y_{s}\end{array}\right)$ corresponds to the last sample point of the modifier, Accept class $\omega_{2}$ if $I>0$ and $\omega_{1}$ if $I=0$.

End

If $I>0$

Assign character to class $\omega_{2}$ (Fig. 1(a)).

End

Locate the sample point $\left(x_{\mathrm{m}}, y_{\mathrm{m}}\right)$ satisfying the relation $x_{\mathrm{m}}=\max _{i>s} x_{i}$.

Define the quantity $\quad r=x_{m}-x_{N}$

If $r \geq \mathcal{E}$ and $y_{N}>y_{b}$

Assign the character to class $\omega_{2}$ (Fig. 1(b)); else

Assign it to class $\omega_{1}$ (Fig.1(c)).

End

Here $\mathcal{E}$ is a threshold, empirically set to 0.07 .

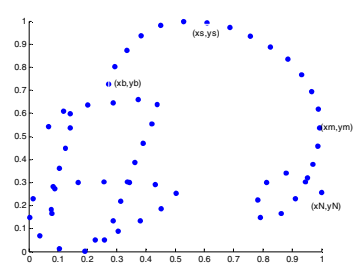

(a)

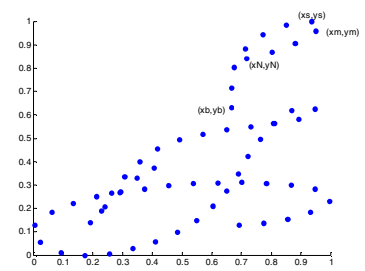

(b)

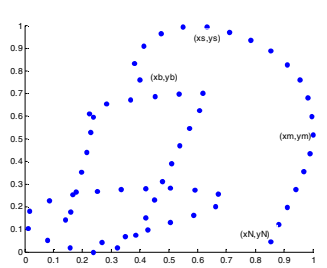

(c)

Fig. 1. Disambiguation of (Group A) confused pairs by structural features 
(a) This sample is assigned to class கீ (by Step 2). Here $I=1 . \quad$ (b) This sample is assigned to கீ (by Step 3). Here $\mathrm{r}>\boldsymbol{\varepsilon}, y_{N}>y_{b}$ and $I=0$ (c) This sample is assigned to கी (by Step 3). Here $\mathrm{r}>\mathcal{E}, y_{N}<y_{b}$ and $I=0$.

\section{Disambiguation of Group B Confusion Pairs}

The discriminative substrokes of confused pairs in Group B may fall in the middle of the trace. Hence, the post processing algorithms for this group must be able enough to automatically extract and analyze parts of strokes that differ. Unlike in Group A, where a single algorithm is used for disambiguation, separate post processing algorithms are generated for each confusion pair in Group B. The need for using dedicated post processing scheme to disambiguate a specific confusion pair arises mainly due to the structural characteristics of the pair under consideration. For example, the post processing scheme for extracting the discriminative sub stroke of the (ன ள) pair will be different from that used for extracting the sub stroke in $(\varphi ு ழ)$ pair. Note that, however, in both these pairs, the finer nuance that makes the confused characters distinct is at the middle of the online trace. In conclusion, for a given pair, the proposed algorithms for Group B take into consideration the temporal information, writing styles and structural cues of the confused characters. The illustrations that follow will throw light on the effectiveness of the methods in both sub stroke extraction and analysis.

A) Consider the characters $ஐ$ and ஜ. Instead of feeding the $(\mathrm{x}, \mathrm{y})$ coordinates of these characters as a whole to the post- processing module, we focus on the shape of sub strokes forming the tails of these characters and extract Fourier descriptors from them. In order to extract the shape of interest, we compute the length of the character and divide it to 4 equal parts (segments). Sample points that lie in the last segment form the tail of the character and are resampled to 30 points before deriving the features. The number of Fourier coefficients chosen is set empirically to 10 . A nearest neighbor classifier is used to obtain the final recognition label of a test character.

B) Consider the characters $ன$ and $ள$. Since the subtle difference in these characters is observed in the middle of the trace, Fourier descriptors do not form a robust feature for discrimination. Our post processing algorithm first automatically extracts the substroke of interest as follows. Let $\left\{\left(x_{i}, y_{i}\right)\right\}_{i=1}^{N}$ be the sample points of preprocessed character (ன оr ள). Locate the first minimum $\left(x_{c}, y_{c}\right)$ that satisfies the condition $y_{c}<y_{c-1}$ and $y_{c}<y_{c+1}$. Starting from $\left(x_{c}, y_{c}\right)$, move along the trace and locate the point $\left(x_{b}, y_{b}\right)$ whose $\mathrm{x}$ coordinate just exceeds $x_{c \text {. }}$ A substroke is extracted with $\left(x_{b}, y_{b}\right)$ as the starting point. The extracted substroke can thus be described as 


$$
S=\left\{\left(x_{i}, y_{i}\right)\right\}_{i=b}^{N} \text {. }
$$

Having extracted the substroke $S$, we shift our focus to analyzing the same. Let $\left(x_{i n t}\right.$, $y_{\text {int }}$ ) be the first encountered minimum in $S$ for which

$$
y_{\text {int }-1}<y_{\text {int }}<y_{\text {int }+1} \text {. }
$$

Fig. 2 provides a pictorial illustration of the aforementioned explanation.

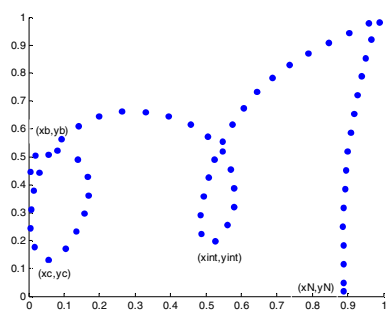

Fig. 2. Illustration of how $\left(x_{i n t}, y_{\text {int }}\right)$ is located in the character व

The following conditions are applied to the point $\left(x_{i n t}, y_{\text {int }}\right)$ for finalizing our decision and thereby resolving the confusion.

1) If $x_{\text {int }-1}<x_{i n t+1}$, assign symbol to ள. (Fig. 3 (a))

2) If the angle between successive pen directions defined at $\left(x_{i n t}, y_{\text {int }}\right)$ is greater than $150 \mathrm{deg}$, assign

symbol to ள. (Fig. 3 (b))

Angle between successive pen directions at $\left(x_{i n t}, y_{\text {int }}\right)$ refers to the angle formed by the vectors

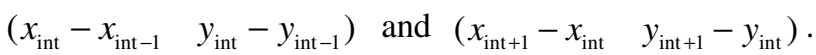

$3)$ If points in the neighborhood of $\left(x_{i n t}, y_{i n t}\right)$ are sufficiently close to each other (less than a threshold), the character is assigned to ள. (Fig. 3 (b))

The 'closeness' condition can be interpreted as follows: Let $W=\left\{\left(x_{i}, y_{i}\right)\right\}_{i=\text { int }-3}^{\text {int } 3}$ be a window size of 7 samples centered at $\left(x_{i n t}, y_{\text {int }}\right)$. Using this window, compute three distances $D_{1}, D_{2}$ and $D_{3}$ as defined below

$$
D_{j}=\operatorname{dist}\left(\left(\begin{array}{ll}
x_{i n t-j} & y_{i n t-j}
\end{array}\right)\left(\begin{array}{ll}
x_{i n t+j} & y_{i n t+j}
\end{array}\right)\right) \quad j=1,2,3
$$

Our final decision for deciding the label of the character can be formulated as follows: Assign character to ள if $\sqrt{D_{1}^{2}+D_{2}^{2}+D_{3}^{2}}<0.1$.

4) If neither condition hold good, character is assigned to ள. (Fig. 3 (c)). 


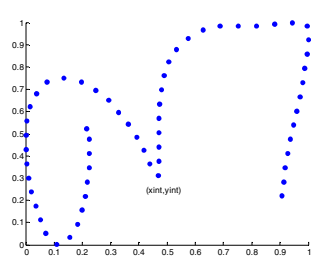

(a)

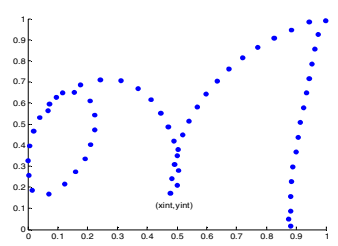

(b)

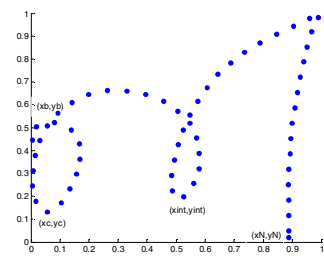

(c)

Fig. 3. (a) (b) indicate samples of $\mathrm{m}$ that satisfy at least one of the conditions (1) (2) (3). Fig. 3 (c) depicts the sample ன that violates the above conditions.

C) We describe the strategy proposed to reduce the confusion between the symbols $(\varphi \varphi, ழ \zeta)$. The intelligence for segmenting out the substroke of interest, $S$ is described as follows. Locate the first sample point $\left(x_{b}, y_{b}\right)$ for which the following 2 conditions are simultaneously satisfied.

$$
\begin{aligned}
& y_{b+1}<y_{b} \text { and } y_{b-1}<y_{b} . \\
& x_{b+1}<x_{b}<x_{b-1} .
\end{aligned}
$$

$\left(x_{b}, y_{b}\right)$ serves as the starting point for our substroke. The minimum y coordinate of the symbol $y_{\min }$ is considered to be the end point. Stated in another

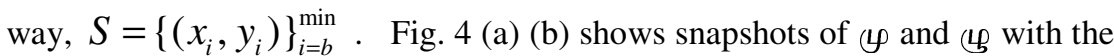
extracted sub strokes.

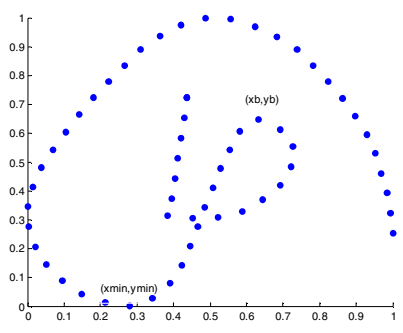

(a)

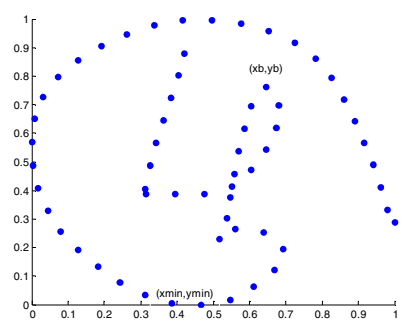

( b )

Fig. 4. (a) (b). Samples of $ம ு$ and ழு with the desired sub strokes respectively.

We now proceed with analyzing the substroke $\mathrm{S}$ as follows. Calculate the number of sample points, $f$ in $\mathrm{S}$ for which $\mathrm{y}_{\mathrm{i}+1}>\mathrm{y}_{\mathrm{i}}$. If $f>0$, the character is assigned to $ழ$. Otherwise $\varphi ு$ is selected. Using this condition, we see that the character in Fig. 4 (a) is assigned to $\varphi$ since $f=0$ for this sample. Fig. 4(b) provides a sample for which $f=1$. Hence, by our logic, it is assigned to ழூ. 


\section{Experimental Results}

The aforementioned techniques are tested on the IWFHR Competition dataset [6]. Table 2 depicts the recognition accuracies obtained by incorporating the post processing scheme for disambiguating confused characters. It can be noted that the ambiguity amongst the symbols have been resolved significantly, as indicated by the substantially high classification rates. The algorithms can also be extended to disambiguate confusion quadruples like (னி ளி னீ ளீ). Here, we first invoke the appropriate post processing algorithms to distinguish the vowel modifiers $\sigma$ and $\rho$ (Group A pair disambiguation) and then discriminate the base consonants disambiguation). Employing the scheme to the quadruple gives a recognition rate of $96.2 \%$. However, it is important mentioning here that the accuracies quoted in Table 2 are applicable only to the confused characters in the pair under consideration. Overall, we have observed an improvement of $1 \%$ in the accuracy of the recognition system. Needless to say, the post processing methods can be applied irrespective of the classifier used for the recognition, though the nature of the confusion matrix may slightly vary.

Table 2. Recognition accuracies after invoking appropriate post processing algorithms to confusion pairs

GROUP A

GROUP B

\begin{tabular}{|l|l|l|l|}
\hline (ஙி ஙீ) & $99.2 \%$ & (ஐ ஜ) & $98.9 \%$ \\
\hline (ணி ணீ) & $98.5 \%$ & (ன ள) & $98.2 \%$ \\
\hline ( ளி ளீ) & $98.1 \%$ & (னி ளி $)$ & $98.3 \%$ \\
\hline (னி னீ) & $97.6 \%$ & (னீ ளீ $)$ & $97.2 \%$ \\
\hline (கி கீ) & $99.1 \%$ & (மு ழு $)$ & $97.5 \%$ \\
\hline
\end{tabular}

In this work, we have designed post processing algorithms to reduce the ambiguity between frequently confused Tamil characters. Structural cues are utilized in the design of these methods, which can be applied independent of the classifier used for the recognition. Future areas of research would involve the utilization of language models as a post processing module in place of structural cues. 
Acknowledgements. The authors would specially like to thank TDIL, DIT for funding this research work.

\section{References}

1. Joshi, N., Sita, G., Ramakrishnan, A.G., Madhavanath, S.: Comparison of Elastic Matching Algorithms for Online Tamil Handwritten Character Recognition. In: Proc. Intl. Workshop Frontiers Handwriting Recog., pp. 444-449 (2004)

2. Deepu, V., Madhavanath, S., Ramakrishnan., A.G.: Principal Component Analysis for Online Handwritten Character Recognition. In: Proc. Intl. Conf. Pattern Recog., vol. 2, pp. 327-330 (2004)

3. Toselli, A.H., Pastor, M., Vidal, E.: On-Line Handwriting Recognition System for Tamil Handwritten Characters. In: Martí, J., Benedí, J.M., Mendonça, A.M., Serrat, J. (eds.) IbPRIA 2007. LNCS, vol. 4477, pp. 370-377. Springer, Heidelberg (2007)

4. Prasad, S.: Online Handwriting Recognition for Tamil. Masters Thesis Report 2008. Indian Institute of Science, Bangalore, India (2008)

5. Sundaram, S., Ramakrishnan, A.G.: Improvement of Online Tamil Character Recognition Engine using Post Processing Methods. Accepted for publication in: Proc. Tenth Intl. Conf. on Doc. Anal. and Recog., ICDAR (2009)

6. HP Labs Isolated Handwritten Tamil Character Dataset, http: / /www.hpl.hp.com/india/research/ penhw-interfaces-1linguistics.html\#datasets 